\title{
Performance Analysis of various Image Watermarking Techniques using different Image Quality Parameters
}

\author{
Sandeep Kumar Jatav \\ IET, DAVV INDORE, \\ INDIA
}

\author{
Dhiiraj Nitnawwre, PhD \\ IET, DAVV INDORE, \\ INDIA
}

\begin{abstract}
Digital technologies is showing tremendous growth and advancements in image transmission and processing during last few years. The image security during image transmission is one of the important areas for research. Various digital watermarking techniques are nowadays become popular for image security and copyright. But, due the rapid growth of the Internet and the development of digital multimedia technologies, illegal copying, modifying and copyright protection have become very important issues. Hence, there is a strong need of development of technique which will able to face these hurdles. There are various techniques to produce the watermark in digital images. In this paper, SVD, DCT, DWT and mixed watermarking techniques has been used for watermarking. The performance parameter like PSNR, MSE, MSSIM and WPSNR are used to evaluate the watermarking performance. The experimental results are shown in this paper for various methods.
\end{abstract}

Keywords: SVD, DCT, DWT, MSSIM, PSNR, WPSNR

\section{INTRODUCTION}

Images are more elucidatory than Words. An image explains the imagination associated with its creation. A Picture portrays the beautiful ideas of its inventor. Here cannot imagine the existence of this world without colorful images be it photo gallery of friends and family, technical images related to studies, artistic images from the brush of an expert etc. It can share these images with friends and colleagues, family and relatives and unknowingly with so many outsiders. The very idea of one's private memories being public is so dreadful. The Internet pace of business today unfortunately leads many to take unethical shortcuts to get the data they want, including stealing it, duplicating it or passing it off as their own original work. Due to the ever growing popularity of internet and explosive number of users worldwide, digital images are less secure nowadays. It is said that every problem has a solution, so is the case of illegal copying of images. Digital watermarking has been proposed as a way to prevent the unauthorized forgery. Its objective is to permanently and unalterably mark the image so that the credit or assignment is farther dispute. In the event of illegal use, the watermark would facilitate the claim of proprietorship, the receipt of copyright revenues, or successful prosecution. The sudden increase in watermarking interest is most likely due to the increase in concern over copyright protection and authentication of content. Copyright-protected digital contents are easily recorded and distributed due to two main factorsprevalence of high-capacity digital recording devices and the explosive growth in using Internet. Watermarking has also been proposed for tracing images that have been illicitly redistributed. In the past, the infraction of large-scale photocopying and distribution often limited copyright infraction, but modern digital networks make large-scale dissemination simple and inexpensive. Digital watermarks are a method to assert an intellectual copyright in the electronic world [3]. The researchers have found some solutions of protecting the digital content of importance, by watermarking, stenography, encryption etc. In this paper discuss the phenomenon of digital watermarking of color images to avoid their misuse online. It has become a very active field of the multimedia security. Watermarking has been studied for several years as one of the widely used copyright protection methods. Schemes of embedding digital watermark into grayscale images have been researched and developed greatly. However, color images are more common in everyday life, and it contains more information than grayscale image and provides more information against the attack of illegal copyright. So it is very important to embed the watermarking into color image for copyright protection.

The effective watermarking technique is one that sustains all kinds of attacks on it without losing its originality. Embedding watermark in spatial domain and frequency domain of the images are the two very popular approaches. Both the ways are accepted and practiced worldwide and have gained prominence due to their positive results.

\section{IMAGE WATERMARKING}

The term "watermark" was probably originated from the German term "wassermarke". The name is probably given because the marks resemble the effects of water on paper. The first paper watermarks did not appear until 1282, in Italy. By the 18th century, watermarks on paper in America and Europe had been used as brands, to record the manufactured date, or to indicate size of original sheets. Digital Watermarking began in 1979, but it was not until 1990 that it gained popularity. The term "digital watermark" was first coined in 1992 by Andrew Tirkel and Charles Osborne [4]. A watermark is very useful in the examination of paper because it can be used for identifying sizes, mill trademarks, locations, and quality or condition of a paper. Encoding an identifying code into digitized text, music, video, picture or other file is known as a digital watermark. A watermark is a name or logo stamped on or included in the makeup of the paper. A few watermarks only appear when people try to copy a document; copies of checks frequently have "void" on the front of them. Traditional watermarks are also utilized on currency to prove that the money is not counterfeit. Digital watermarks are the electronic versions of their traditional counterpart.

Digital watermarking is the process of computer-aided information hiding in a carrier signal; the hidden information should but does not need to contain a relation to the carrier signal. Digital watermarks mainly used to verify the authenticity or integrity of the carrier signal or to show the identity of its owners. 


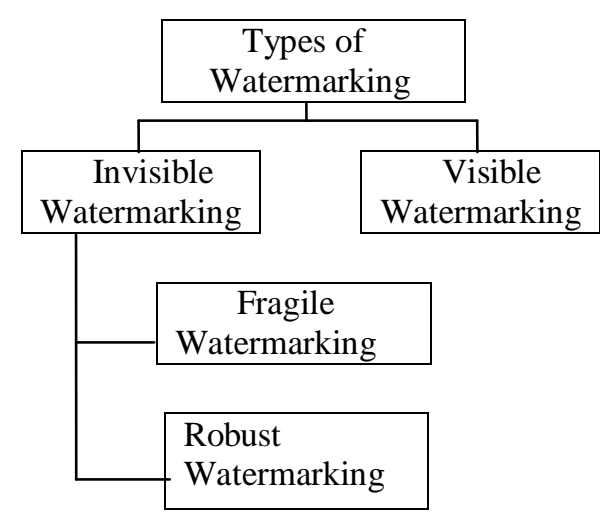

Fig 1: Types of watermarking

In general, digital watermarking has following features-

1. Perceptual transparency: The algorithm embeds data quality of the underlying host signal without affecting the perceptual quality of the underlying host signal.

2. Security: A secure data embedding procedure cannot be broken unless the unauthorized user access to a secret key that controls the insertion of the data in the host signal.

3. Robustness: The digital watermark must survive after being attacked by lossy data compression and image manipulation and processing operations, e.g. cut and paste, filtering etc.

4. Unambiguous: Retrieval of the watermark should unambiguously identify the owner.

5. Imperceptibility: The watermark itself should not be visible by the human visual system (HVS) and should not degrade the image quality.

6. Reliability: To ensure that the project application returns the correct watermark each time.

There is various application of water marking like copyright protection, Content authentication, Fingerprinting, Broadcast monitoring etc.

\section{DIGITAL WATERMARKING TECHNIQUES}

\subsection{SVD based Watermarking}

SVD is one of the effective tool to analysis the matrices. Suppose that the input image is represented by a matrix A. For convenience, assume that $\mathrm{A}$ is an $\mathrm{N} \times \mathrm{N}$ square matrix with rank $r$. The SVD of A can be represented by

$$
\mathrm{A}=\mathrm{U}^{*} \mathrm{D}^{*} \mathrm{~V}^{\mathrm{T}}
$$

Where $\mathrm{U}$ and $\mathrm{V}$ are $\mathrm{N} \cdot \mathrm{N}$ orthogonal matrices and $\mathrm{D}$ is an $\mathrm{N}$ by $\mathrm{N}$ singular, diagonal matrix with singular values diagonal entries [5]. While using the SVD transformation a matrix is decomposed into three matrices $\mathrm{U}, \mathrm{D}, \mathrm{V}$. $\mathrm{U}$ and $\mathrm{V}$ are the unitary matrices and $\mathrm{D}$ is a diagonal matrix.

\subsection{DWT based Watermarking}

Wavelet transform has been widely studied in signal processing in general and image compression in particular. Here DWT2 (Two dimensional Discrete Wavelet Transform) method is used to decompose the image into four sub bands or components namely LL, LH, HL \& HH.

LL-Low frequency band

LH-Horizontal high frequency band

HL-Vertical high frequency band
HH-Diagonal high frequency band

Wavelet coding algorithms are especially suitable for applications where scalability and tolerable degradation are important [6].

\begin{tabular}{|l|l|}
\hline$L L_{1}$ & $H L_{1}$ \\
\hline$L H_{1}$ & $H H_{1}$ \\
\hline
\end{tabular}

Fig 2: Single level Decomposition using DWT

\section{- Characteristics of DWT}

1) The wavelet transform decomposes the image into three spatial directions i.e. diagonal, horizontal and vertical. Hence wavelets are reflect the anisotropic properties of HVS more precisely.

2) Watermark detection at lower resolutions level is computationally effective because at every successive resolution level there are few frequency bands involved.

3) As LL band contains largest wavelet coefficients, scale factor is chosen accordingly upto 0.05 value for $\mathrm{LL}$ and 0.005 values for other different bands. For this pair of values, there is no degradation in watermarked image.

4) High resolution sub bands helps to easily locate edge and textures patterns in an image [7].

\subsection{DCT Based Watermarking}

The discrete cosine transforms is a technique for converting a signal into elementary frequency domain signal. It represents an image as a sum of sinusoids of varying magnitudes and frequencies. With an input image " $x$ " the DCT coefficients for the transformed output image " $y$ " are computed according to Equation. 1 shown below. In the equation " $x$ " is the input image having $\mathrm{N} \times \mathrm{M}$ pixels, $\mathrm{x}(\mathrm{m}, \mathrm{n})$ is the intensity of the pixel in row $\mathrm{m}$ and column $\mathrm{n}$ of the image, and $\mathrm{y}(\mathrm{u}, \mathrm{v})$ is the DCT coefficient in row $\mathrm{u}$ and column $\mathrm{v}$ of the DCT matrix.

$$
\begin{aligned}
\mathrm{y}(\mathrm{u}, \mathrm{v}) & =\sqrt{\frac{2}{M}} \sqrt{\frac{2}{N}} \propto_{m} \propto_{n} \sum_{x=0}^{M-1} \sum_{y=0}^{N-1}\{x(m, n) \\
& \left.\times \cos \frac{(2 m+1) u \pi}{2 M} \cos \frac{(2 n+1) u \pi}{2 N}\right\}
\end{aligned}
$$

Where

$$
\begin{aligned}
& \propto_{u}=\left\{\frac{1}{\sqrt{2}}\right. \\
& u=0 \\
& \{1 \quad u=1,2,3 . \ldots . M-1
\end{aligned}
$$

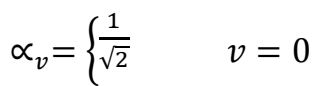

$$
\begin{aligned}
& \{1 \quad v=1,2,3 \ldots \ldots \ldots N-1
\end{aligned}
$$


The image is reconstructed by applying inverse DCT operation according to Equation. 2:

$$
\begin{aligned}
x(m, n)= & \sqrt{\frac{2}{M}} \sqrt{\frac{2}{N}} \sum_{u=0}^{M-1} \sum_{v=0}^{N-1}\left\{\propto_{m} \propto_{n} y(u, v)\right. \\
& \left.\times \cos \frac{(2 m+1) u \pi}{2 M} \cos \frac{(2 n+1) u \pi}{2 N}\right\}
\end{aligned}
$$

The popular block based DCT transform segments image nonoverlapping blocks and applies DCT to each block. These results in giving three frequency coefficient bands: mid frequency sub band, low frequency sub band and high frequency sub band. DCT based watermarking is based on the two facts [11]. The first fact is that the much of signal energy lies at low frequencies sub band which contains the most important visual parts of the image. The second fact is that the high frequency components of the image are usually removed through compression and various noise related attacks. The watermark is embedded by modifying the coefficients of the middle frequency sub band so that the visibility of the image will not be affected and the watermark will not be removed by compression [8].

\subsection{SVD-DCT Based Watermarking}

Robustness, capacity and imperceptibility are the three important requirements of an efficient watermarking scheme. SVD based watermarking scheme has high imperceptibility. Although SVD based scheme withstands using different image quality measures. Also SVD based technique has only limited capacity [9]. These limitations have led to the development of a new scheme that clubs the properties of SVD and DCT. This particular algorithm proves to be better than ordinary SVD based watermarking and ordinary DCT based watermarking scheme.

\subsection{SVD-DWT Based Watermarking}

Here above mentioned SVD-DCT scheme has enormous capacity because data embedding is possible in all the sub bands. Watermark was achieved good imperceptibility. Disadvantage is that the embedding and the recovery are time consuming process because the zigzag scanning to map the coefficients into four quadrants based on the frequency. Alternatively if we apply DWT we get the four frequency sub bands directly namely; approximation, diagonal, horizontal and vertical bands. So the time consumption will be greatly reduced [9]. Also, SVD is a very convenient tool for watermarking in the DWT domain.

\subsection{SVD-DCT-DWT Based Watermarking}

This method utilizes the wavelet coefficients of the cover image to embed the watermark. Any of the three high frequency sub bands of wavelet coefficients can be used to watermark the image. The DCT coefficients of wavelet coefficients are calculated and singular values decomposed. The singular values of the cover image and watermark are added to form the modified singular values of the watermarked image. Then the inverse DCT transform is applied followed by the inverse DWT. This is algorithm that clubs the properties of SVD, DCT and DWT [9]. Watermark embedded using this algorithm is highly imperceptible. This scheme is robust using different kinds of image quality parameters such as PSNR WPSNR and MSE. It has very high data hiding capacity.
The new method was found to satisfy all the requisites of an ideal watermarking scheme such as imperceptibility or fidelity, robustness and good capacity. This method can be used for authentication and data hiding purposes.

\section{WATERMARK EMBEDDING ALGORITHM}

Let ' $A$ ' be the cover image. Apply DWT to decompose the image into four sub-bands LL, HL, LH and HH. Take any of these four sub-bands. Apply DCT to the chosen sub-band. Let ' $B$ ' denote the matrix obtained after applying DCT. Now $B$ acts as the host image. Apply SVD so that ' $B$ ' can then be written as $B=U_{\mathrm{B}} \Sigma \mathrm{B}_{\mathrm{B}} V \mathrm{~B}_{\mathrm{B}}^{\mathrm{T}}$ where $U_{\mathrm{B}}$ and $V_{\mathrm{B}}^{\mathrm{T}}$ are the orthonormal unitary matrices of $B$. The term $\Sigma_{\mathrm{B}}$ constitutes the singular values of the matrix of $B$.

Let ' $W$ ' represent the watermark. Apply DWT and take any of the four sub-bands. Apply DCT to the chosen sub-band. Let ' $S$ ' denote the matrix obtained after applying DCT. Now $B$ acts as the host image. Apply SVD so that ' $S$ ' can then be written as $S=U_{\mathrm{S}} \Sigma_{\mathrm{S}} V_{\mathrm{S}}^{\mathrm{T}}$ where $U_{\mathrm{S}}$ and $V_{\mathrm{S}}^{\mathrm{T}}$ are the orthonormal unitary matrices of $S$. The term $\Sigma_{S}$ constitute the singular values of the matrix $S$. Modify singular values of $B$ using singular values of $S$. Then perform IDCT followed by IDWT to obtain the watermarked image. The four sets of DWT coefficients can be used to embed four different visual watermarks or the same watermark.

\section{WATERMARK EXTRACTION ALGORITHM}

Let 'A' be the cover image. Apply DWT and take any of the four sub-bands. Apply DCT to the chosen sub-band. Let ' $B$ ' denote the matrix obtained after applying DCT. Now $B$ acts as the host image. Apply SVD so that ' $B$ ' can then be written as $B=U_{\mathrm{B}} \Sigma_{\mathrm{B}} V_{\mathrm{B}}^{\mathrm{T}}$ where $U_{\mathrm{B}}$ and $V_{\mathrm{B}}^{\mathrm{T}}$ are the orthonormal unitary matrices of $B$. Term $\Sigma_{\mathrm{B}}$ constitutes singular values of the matrix of $B$.

Let ' $\mathrm{W}^{*}$ ' be the watermarked image. Apply DWT and take any of the four sub-bands. Apply DCT to the chosen subband. Let ' $A$ '' denote the matrix obtained after applying DCT. Now $A^{*}$ acts as the host image. Apply SVD so that ' $B$ ' can then be written as $B=U_{\mathrm{A}^{*}} \Sigma_{\mathrm{A}^{*}} \mathrm{~V}_{\mathrm{A}^{*}}{ }^{\mathrm{T}}$ where $U_{\mathrm{A}^{*}}$ and $V_{\mathrm{A}^{*}}{ }^{\mathrm{T}}$ are the orthonormal unitary matrices of $A^{*}$. Term $\Sigma_{\mathrm{A}^{*}}$ constitutes the singular values of the matrix of $A^{*}$. Watermark is extracted by subtracting the singular values obtained above.

\section{EXPERIMENTAL RESULT}

In this paper, We have taken two $512 \times 512$ gray scale image are used as cover image and watermark image respectively. Figures show that the watermarked image and extracted watermark is robust using different Image quality parameters. Tables show the comparison between earlier SVD, DCT, DWT, DCT-SVD, DWT-SVD and SVD-DCT-DWT methods. Higher the value of PSNR, it is hard to be aware of the differences with the cover image by human eye system. When watermark is embedded in textured region and into edges, WPSNR is adequate to calculate. Value of WPSNR will be slightly higher than PSNR to reflect the fact that human eye will have less sensitivity to modifications in textured areas than in smooth areas. Also, value of MSSIM is close to 1 which is required. 


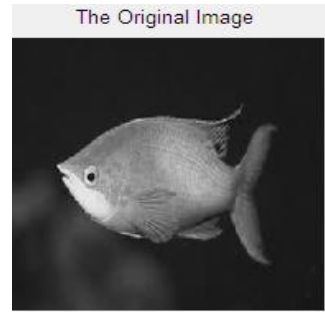

Fig 3: (a)

The Waterma-ked Imace

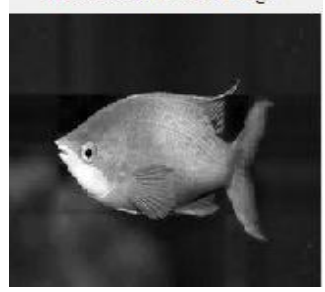

Fig 3: (c)

The Watermarked Image

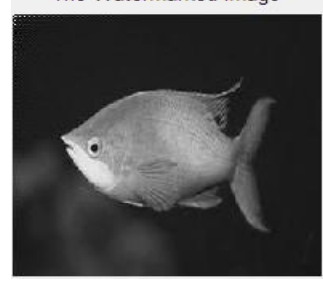

Fig 3: (e)

The Watermarked lmage

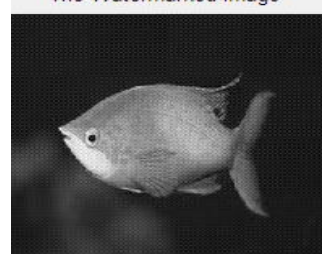

Fig 3: (g)

The Watermarked Image

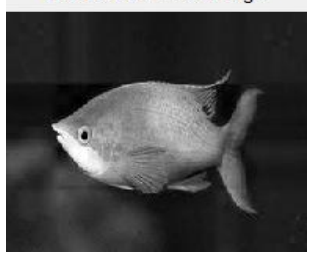

Fig 3: (i)

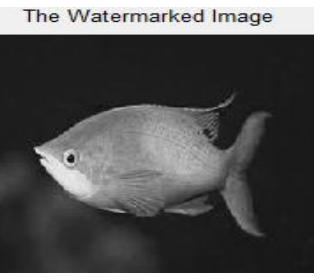

Fig 3: (k)

The Watermarked Image

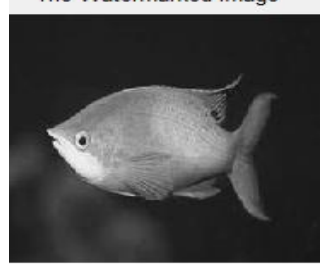

Fig 3: (m)

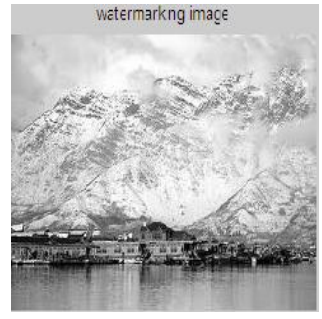

Fig 3: (b)

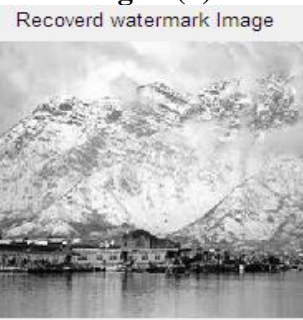

Fig 3: (d)

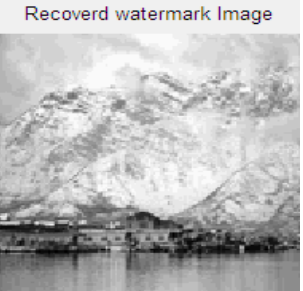

Fig 3: (f)

Recoverd watermark Image

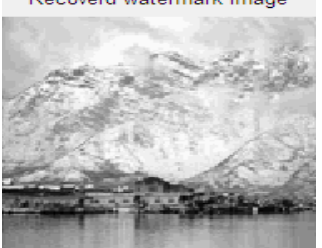

Fig 3: (h)

Recoverd watermark Image

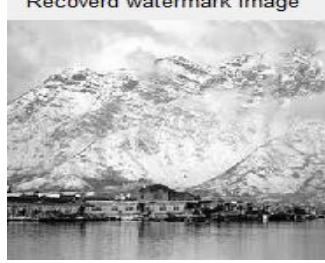

Fig 3: (j)

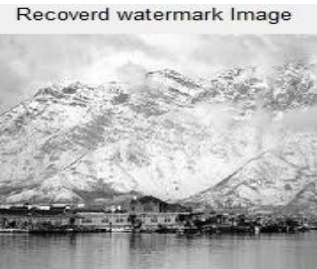

Fig 3: (l)

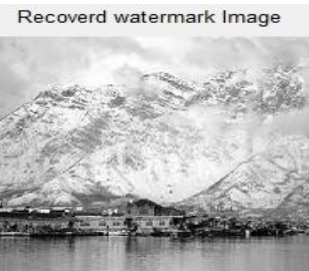

Fig 3: (n)

Fig 3: Images of different algorithm

(a) Original image (b) Watermark image (c) Embedded watermark image using SVD (d) Extracted watermark image using SVD (e) Embedded watermark image using DCT (f) Extracted watermark image using DCT (g) Embedded watermark image using DWT (h) Extraction watermark image using DWT (i) Embedded watermark image using SVD-DCT (j) Extraction watermark image using SVD-DCT (k) Embedded watermark image using SVD-DWT (l) Extraction watermark image using SVD-DWT (m) Embedded watermark image using SVD-DCT-DWT (n) Extraction watermark image using SVD-DCT-DWT

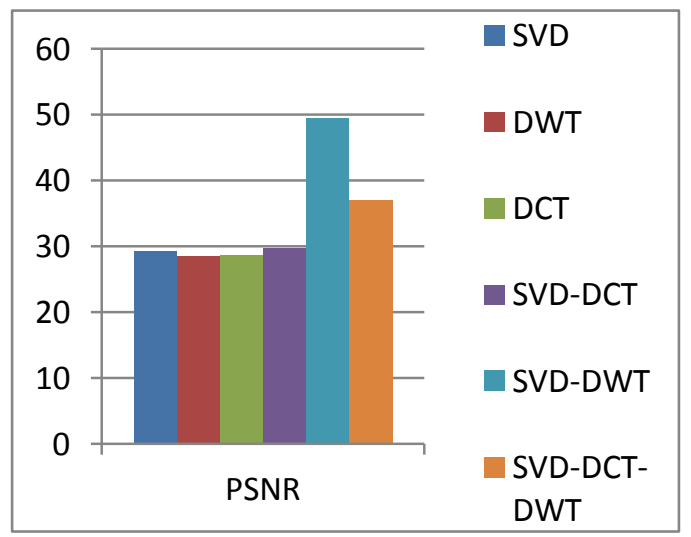

Fig 4: Graph showing PSNR values of different algorithm for Embedded watermark

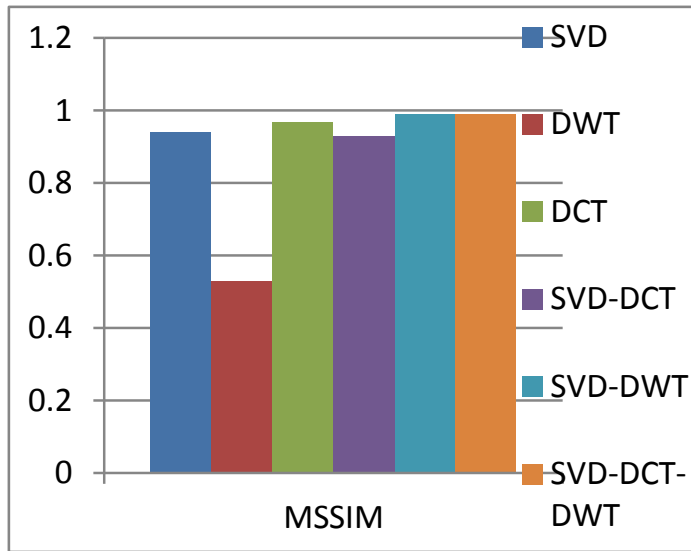

Fig 5: Graph showing MSSIM values of different algorithm for Embedded watermark

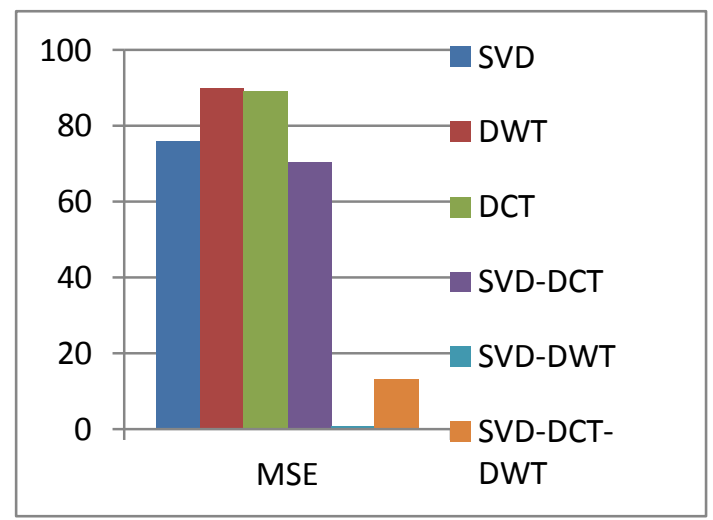

Fig 6: Graph showing MSE values of different algorithm for Embedded watermark 


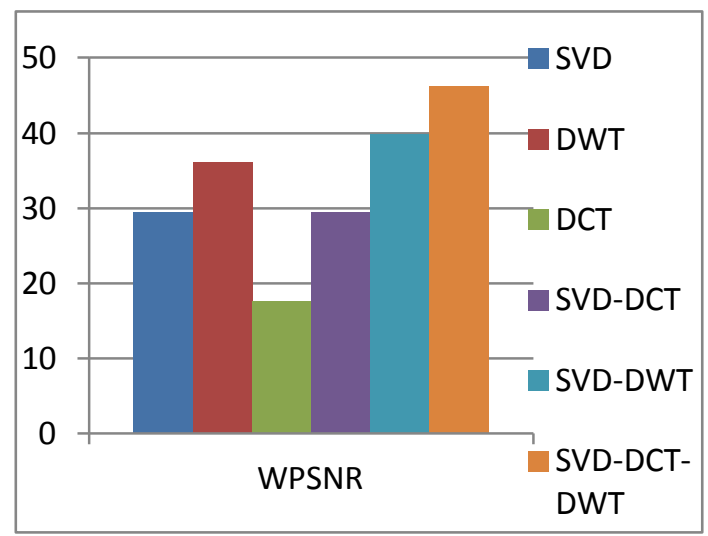

Fig 7: Graph showing WPSNR values of different algorithm for Embedded watermark

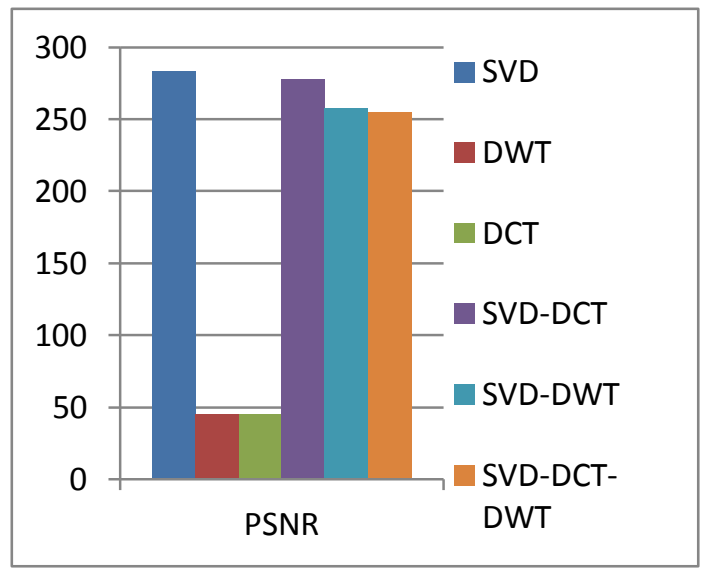

Fig 8: Graph showing PSNR values of different algorithm for Extraction watermark

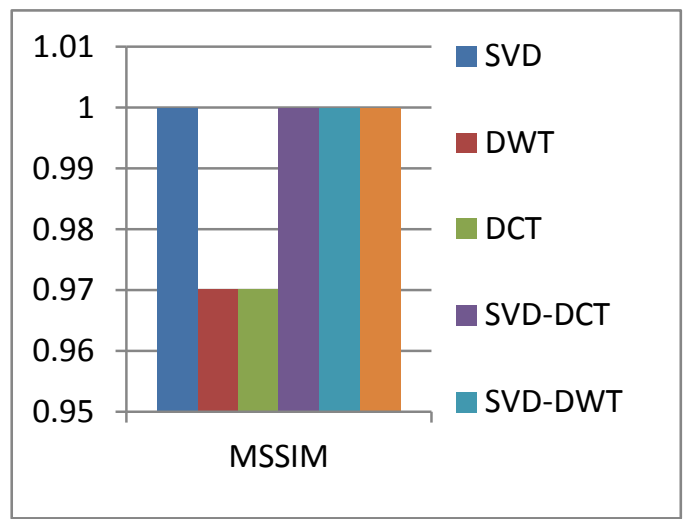

Fig 10: Graph showing MSSIM values of different algorithm for Extraction watermark

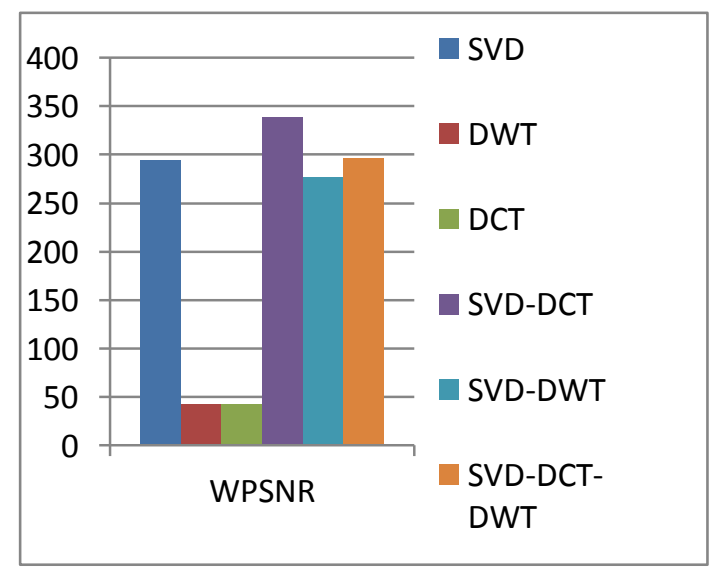

Fig 11: Graph showing WPSNR values of different algorithm for Extraction watermark

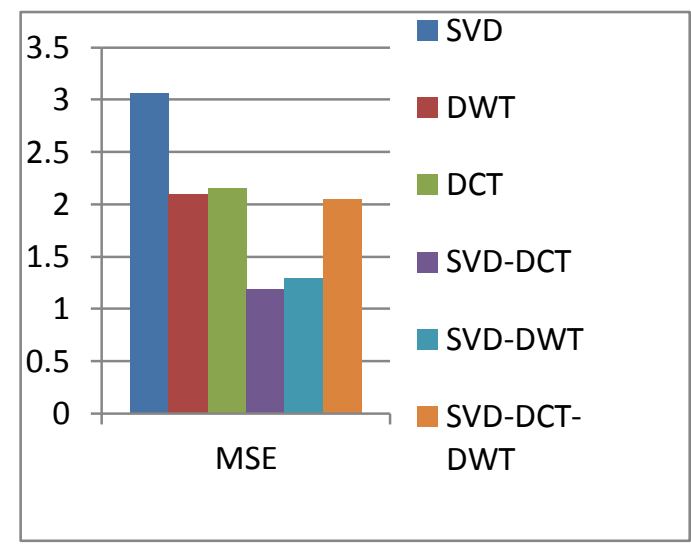

Fig 9: Graph showing MSE values of different algorithm for Extraction watermark 
Table1. Parameters value for different Algorithm

\begin{tabular}{|l|c|c|c|c|c|}
\hline \multicolumn{2}{|l}{ ALGORITHMS $\downarrow$} & \multicolumn{2}{c|}{ IMAGE QUALITY PARAMETERS } \\
\cline { 3 - 6 } \multicolumn{2}{|c|}{} & PSNR (dB) & MSE & MSSIM & $\begin{array}{l}\text { WPSNR } \\
(\mathbf{d B})\end{array}$ \\
\hline \multirow{3}{*}{ SVD } & EMBEDDED WATERMAK & 29.3244 & 75.9694 & 0.9417 & 29.0433 \\
\cline { 2 - 6 } & EXTRACTION WATERMARK & 283.2724 & 3.0608 & 1.0000 & 293.6609 \\
\hline \multirow{3}{*}{ DCT } & EMBEDDED WATERMARK & 28.6311 & 89.1192 & 0.9768 & 17.6055 \\
\cline { 2 - 6 } & EXTRACTION WATERMARK & 44.7867 & 2.1598 & 0.9762 & 42.9446 \\
\hline \multirow{3}{*}{ DWT } & EMBEDDED WATERMARK & 28.5951 & 89.8611 & 0.5344 & 36.1216 \\
\cline { 2 - 6 } & EXTRACTION WATERMARK & 44.8982 & 2.1055 & 0.97626 & 42.9446 \\
\hline \multirow{2}{*}{ SVD-DWT } & EMBEDDED WATERMAK & 29.6491 & 70.4968 & 0.9347 & 29.3211 \\
\cline { 2 - 6 } & EXTRACTION WATERMARK & 277.3423 & 1.1991 & 1.0000 & 338.8678 \\
\cline { 2 - 6 } SVD- DCT- DWT & EMBEDDED WATERMARK & 49.4707 & 0.7345 & 0.9982 & 39.7744 \\
\cline { 2 - 6 } & EXTRACTION WATERMARK & 257.0199 & 1.2917 & 1.0000 & 276.9433 \\
\hline
\end{tabular}

\section{CONCLUSION}

The SVD, DCT and DWT based watermarking scheme has been simulated in this project. The mixed methods like SVDDCT, SVD-DWT and SVD-DCT-DWT are also performed to analysis the mixing effect. The MSE, PSNR, MSSIM and WPSNR parameters are used for performance evaluation of various methods. From the simulation result, We are conclude that:

\section{For Embedded watermark:}

- In case of SVD-DWT, PSNR is high due to the use of only low frequency Components for embedding. High frequency component like edge are preserve.

- Structural symmetry is better in the SVD-DWT method due to high frequency component reproduction of DWT.

The error in low intensity value does not have significant importance. Hence the WPSNR is high for SVD-DCT-DWT where as MSE is low for SVD-DWT.

\section{For Extraction watermark:}

- Due to watermark image available on the receiver side the PSNR is high in the SVD based method. In the distortion less transmission, the error in the extraction part of SVD is $\sim 0$. Due to combining of other methods the error will increased and PSNR get poor compare to SVD. But the MSSIM is 1 for all combination with SVD.

- WPSNR is achieving high in the SVD-DCT due to weighting factor and using the almost null amplitude frequency in the Embedding. Hence this data are recovered maximum.
All above methods were found to satisfy all the requisites of an ideal watermarking scheme such as imperceptibility or fidelity, robustness and good capacity. These methods can be used for authentication and data hiding purposes.

\section{REFERENCES}

[1] W. H. Lin, Y. R. Wang, S. J. Horng, T. W. Kao, and Y. Pan, "A blind watermarking method using maximum wavelet coefficient quantization," Journal of Expert Systems with Applications, vol. 36, pp. 11509-11516, 2009.

[2] Lu, C. S., Huang, S.-K., Sze, C.-J., Liao, H.-Y., "A new watermarking technique for multimedia protection," in Multimedia Image and Video Processing, L. Guan, S.Y. Kung, and J. Larsen, Eds. Boca Raton, FL: CRC, 2001, pp. 507--530.

[3] Cox, IJ, Miller, ML \& Bloom, JA 2002, Digital Watermarking, Morgan Kaufmann Publisher, San Francisco, CA, USA.

[4] A.Z. Tirkel, G.A. Rankin, R.M. Van Schyndel, W.J. Ho, N.R.A. Mee, C.F. Osborne. "Electronic WaterMark". DICTA 93, Macquarie University. P.666673.

[5] V. Gorodetski, L.J. Ipopyack, and V. Samoilov, "SVDbased approach to transparent embedding data into digital images," in Proc. Int. Workshop, MMM-ACNS, St. Petersburg, Russia, 2001, pp. 263-274.

[6] V.M. Potdar, S. Han and E. Chang, A survey of digital image watermarking techniques, in: Proceedings of the IEEE 3rd International Conference on Industrial Informatics (INDIN), Perth, Australia, 10-12 August 2005, pp. 709-716. 
[7] Tao, P \& Eskicioglu, AM 2004, 'A Robust Multiple Watermarking Scheme in the Discrete Wavelet Transform Domain', in Symposium on Internet Multimedia Management Systems V, Philadelphia, PA

[8] Tao, B., Dickinson, B., “Adaptive Watermarking in DCT Domain", in Proc. IEEE International Conference on Acoustics, Speech, and Signal Processing, ICASSP '97, 1997, Vol.4, pp.1985-2988.

[9] S. Murty. P, M. U. Bhaskar, P. N. Babu and P. R. Kumar, "A Semi-Blind Reference Watermarking SchemeUsing DWT-DCT-SVD for Copyright Protection", International Journal of Computer Science \&Information Technology (IJCSIT) vol. 4, no 2, pp. 6982 April 2012.
[10] Kundur D. Hatzinakos, "Digital Watermarking using Multiresolution Wavelet Decomposition", Proc. IEEE Int. Conf. On Acoustics, Speech and Signal Processing, Seattle, Washington, vol. 5, pp. 2969-2972, May 1998.

[11] Choi, Y., Aizawa, K., "Digital Watermarking Technique using Block Correlation of DCT Coefficients" in Electronics and Communica-tions, Japan, Part 2, vol. 85 , no. 9, 2002

[12] Suhail, M., A., Obaidat, M., S., "Digital Watermarking Based DCT and JPEG Model", in IEEE Transactions on Instrumentation and Measurement, Vol. 52, No. 5, Oct. 2003. 